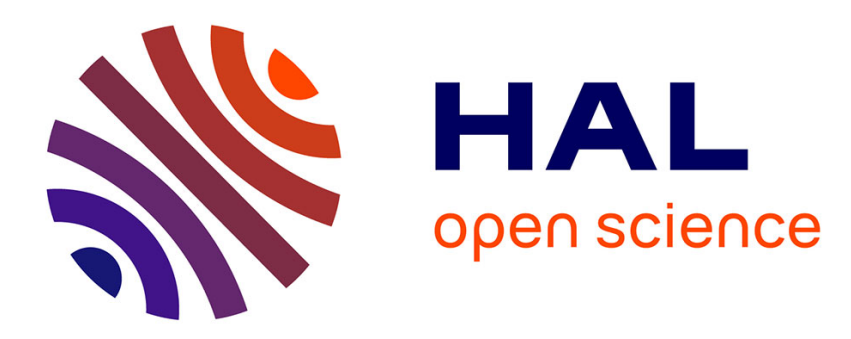

\title{
Les apports de la cytogénétique chez le Porc
}

P. Popescu

\section{- To cite this version:}

P. Popescu. Les apports de la cytogénétique chez le Porc. Productions Animales, 1992, hs (hs), pp.277-280. hal-00896031

\section{HAL Id: hal-00896031 \\ https://hal.science/hal-00896031}

Submitted on 1 Jan 1992

HAL is a multi-disciplinary open access archive for the deposit and dissemination of scientific research documents, whether they are published or not. The documents may come from teaching and research institutions in France or abroad, or from public or private research centers.
L'archive ouverte pluridisciplinaire HAL, est destinée au dépôt et à la diffusion de documents scientifiques de niveau recherche, publiés ou non, émanant des établissements d'enseignement et de recherche français ou étrangers, des laboratoires publics ou privés. 


\section{P. POPESCU}

INRA Laboratoire de Cytogénétique 78352 Jouy-en-Josas Cedex
Apports actuels et futurs des

marqueurs génétiques dans

l'amélioration des populations

animales

Les apports de la cytogénétique chez le Porc

Résumé. Les verrats ayant conçu des portées de petite taille sont détectés au Centre de Traitement de l'Information Général de l'INRA (CTIG) selon un procédé dérivé du programme national de Gestion technique des troupeaux de truies. Les verrats qui engendrent moins de 8 porcelets nés totaux en moyenne par portée, sur au moins 6 mise bas sont considérés hypoprolifiques et leur caryotype est examiné. Depuis 1979 neuf translocations originales ont été découvertes chez des verrats hypoprolifiques.

Chez la truie, les translocations n'ont pas d'effet sur l'intervalle sevrage-oestrus ni sur le taux d'ovulation. Par contre l'intervalle sevrage-fécondation est significativement augmenté.

La réduction de la taille des portées des verrats porteurs est provoquée par la formation de gamètes à caryotype déséquilibré qui fécondent mais produisent des embryons non viables.

Les anomalies chromosomiques peuvent être utilisées comme marqueurs dans les travaux de cartographie génique et pour le tri de chromosomes par la cytométrie en flux.

Nous avons abordé la cytogénétique du Porc vers le milieu des années soixante-dix. En 1979, nous avons décrit pour la première fois en France, une translocation réciproque chez un verrat qui engendrait des portées dont la taille était réduite de moitié et qualifié pour cela "hypoprolifique" (Popescu et Legault 1979).

Dans cette brève mise au point nous nous proposons de décrire les étapes de la détection des verrats "hypoprolifiques", de discuter les effets des translocations sur les performances de reproduction et d'évoquer quelques prolongements vers des recherches plus avancées.

Tableau 1. Exemple de repérage d'un verrat "hypoprolifique" au sein d'un élevage soumis au programme national de gestion technique des troupeaux de truies $\left(^{*}\right)$.

Eleveur : $\operatorname{Mr} X \ldots$

Adresse :

\begin{tabular}{|c|c|c|c|}
\hline \multirow{2}{*}{$\mathrm{N}^{\circ}$ VERRAT } & \multirow{2}{*}{ Nbre de portées } & \multicolumn{2}{|c|}{ Nombre moyen de porcelets nés par portée } \\
\cline { 3 - 4 } & & Moyenne brute & Moyenne corrigée \\
\hline $\mathrm{A}$ & 14 & 10,7 & 11,0 \\
$\mathrm{~B}$ & 15 & 5,1 & 5,8 \\
$\mathrm{C}$ & 13 & 11,6 & 12,1 \\
$\mathrm{D}$ & 13 & 11,9 & 12,6 \\
$\mathrm{E}$ & 13 & 11,3 & 12,0 \\
$\mathrm{~F}$ & 8 & 12,0 & 12,3 \\
\hline
\end{tabular}

* Afin d'éviter toute exploitation abusive, l'anonymat a été préservé pour l'élevage et les verrats en service.

\section{1 / La détection informatisée des verrats "hypoprolifiques"}

Les verrats ayant conçu des portées de taille anormalement faible sont repérés périodiquement au Centre de Traitement de l'Information Général de I'INRA (CTIG) selon un procédé relativement simple dérivé du programme national de Gestion Technique des Troupeaux de Truies (GTTT) décrit par Legault $e t$ al (1971) et par Dagorn (1975).

La prospection s'applique à l'ensemble des élevages soumis à ce programme et à toutes les portées dont le verrat père est correctement identifié. Les verrats sont considérés comme suspects lorsqu'ils ont engendré moins de 8 porcelets nés totaux en moyenne par portée sur un effectif minimum de 6 mises bas. De plus les moyennes corrigées pour l'effet de l'âge à la mise bas (Legault et Owen 1976) sont également fournies pour éviter une éventuelle confusion entre cet effet et celui du verrat. Enfin, comme l'illustre le tableau 1, les informations sont présentées en regard de celles relatives aux verrats contemporains du même élevage.

En faisant l'hypothèse de normalité de la distribution de la taille de la portée à la naissance (moyenne générale $X=10,7$ et écart-type $S=2,7$ ) et en supposant que les variations entre troupeaux sont négligeables, on peut avoir un ordre de grandeur de la probabilité d'obtenir un verrat ayant engendré moins de 8 porcelets par portées en $\mathrm{N}$ mises bas (figure 1 ). Ainsi, pour $\mathrm{N}=12$, il est normal de trouver un verrat "hypoprolifique" sur 4167. Selon un sondage récent portant sur un ensemble de 9570 verrats ayant 
Figure 1.

Principe

de la

détection

de verrats

"hypo-

prolifiques".

\section{$N \quad 6: \bar{X}<8$}

- Du 01/07/86 au 30/06/87: 100 "hypoprolifiques" sur 9570.

- En 1985 : $540^{7}$ "hypoprolifiques" dont $360^{7}$ différents.

- Nb. moy. de portées conçues / verrat "hypoprolifique" : $\bar{N}_{H}=13,7$.

- Nb. moy. de portées conçues / verrat témoin : $\overline{\mathrm{N}}_{\mathrm{T}}=18,5$.

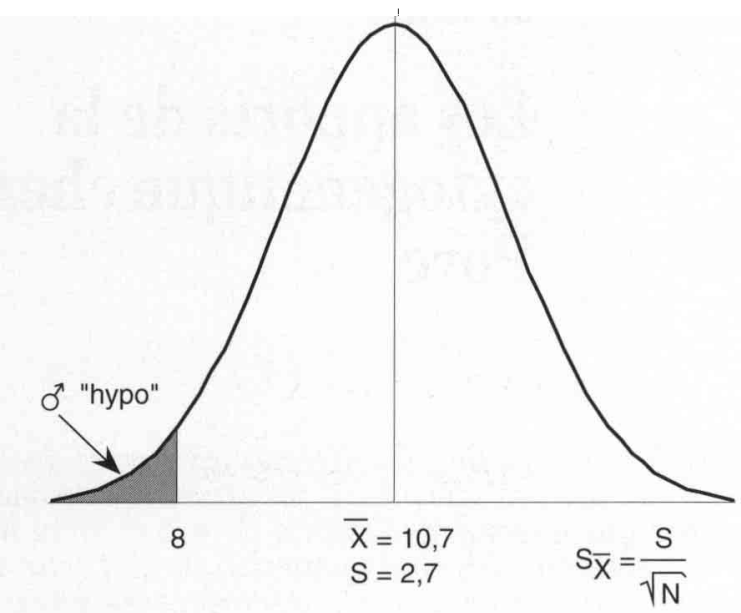

Tableau 2. Probabilité d'obtenir un verrat ayant engendré moins de 8 porcelets par portée en $(N)$ mise bas dans une hypothèse de normalité de la distribution de la taille de la portée.

Moyenne générale : $X=10,7$ porcelets

Ecart-type : $s=2,7$ porcelets

\begin{tabular}{|c|c|c|}
\hline \multirow{2}{*}{ Nombre de portées N } & \multicolumn{2}{|c|}{$\begin{array}{c}\text { Probabilité d'obtenir un verrat "hypoprolifique" } \\
(\mathrm{X}<8)\end{array}$} \\
\cline { 2 - 3 } & Probabilité & Proportion \\
\hline 9 & 0,00135 & 1 sur 741 \\
12 & 0,00024 & 1 sur 4.167 \\
16 & 0,000032 & 1 sur 31.250 \\
\hline
\end{tabular}

engendré plus de 5 portées (21 en moyenne), 10 d'entre eux se sont révélés "hypoprolifiques", proportion qui dépasse très largement les prévisions théoriques et qui laisse supposer l'existence de phénomènes perturbateurs de la distribution normale.

Le même animal peut apparaître au cours de plusieurs sondages successifs. Ainsi, sur l'ensemble de l'année 1985 , 54 cas de verrats hypoprolifiques concernant 36 animaux différents ont été enregistrés ; ils avaient conçu 13,7 portées en moyenne contre 17,5 pour les 198 verrats contemporains des mêmes élevages.

Le système de repérage des verrats "hypoprolifiques" dans les élevages comporte certaines imperfections qui méritent d'être soulignées :

- Le relevé de l'identification du verrat père de la portée n'étant pas indispensable au calcul des paramètres techniques du troupeau, il est généralement considéré comme facultatif par les éleveurs de l'étage de production. Il en résulte que près de $60 \%$ de l'information potentielle est absente. En outre, $25 \%$ environ des verrats n'ont pas produit le nombre minimum de 6 portées. Par conséquent, le programme de détection ne s'applique effectivement qu'à environ $30 \%$ des verrats en service.
- En raison de la généralisation de la "conduite en bandes" les saillies ont lieu d'une manière groupée (sur une période de 2 à 3 jours), la saillie étant généralement répétée deux fois, à 12 ou 24 heures d'intervalle. Or, il arrive assez fréquemment que le verrat ayant effectué la première saillie se trouvant indisponible pour la seconde est remplacé par un autre animal, pratique qui est parfaitement compatible avec l'objectif de l'éleveur de l'étage de production qui est avant tout d'obtenir une portée. Dans les élevages où plusieurs verrats sont en service simultanément (cas le plus répandu) il existe donc une imprécision sur certaines paternités d'autant plus difficiles à déceler que l'espace réservé à l'idendification ne prévoit que l'encodage d'un seul verrat. Cette situation est illustrée par l'histogramme de la figure 2 correspondant à la translocation 4/15. La translocation 4/15 est apparue récemment dans un troupeau où la qualité des enregistrements a permis, en collaboration avec l'éleveur, de distinguer l'effet réel du verrat de l'effet apparent estimé par la GTTT (figure 2). Dans ce cas particulier, l'effet défavorable de la translocation se trouve sous-estimé de 0,95 porcelets par portée.

Depuis 1979, neuf translocations orginales ont été découvertes en France, l'une d'entre elles ayant été observée 6 fois.

Figure 2. Distribution des tailles de portées engendrées par le verrat porteur de la translocation 4-, 15+ avant et après élimination des saillies doubles.

Nombre de portées

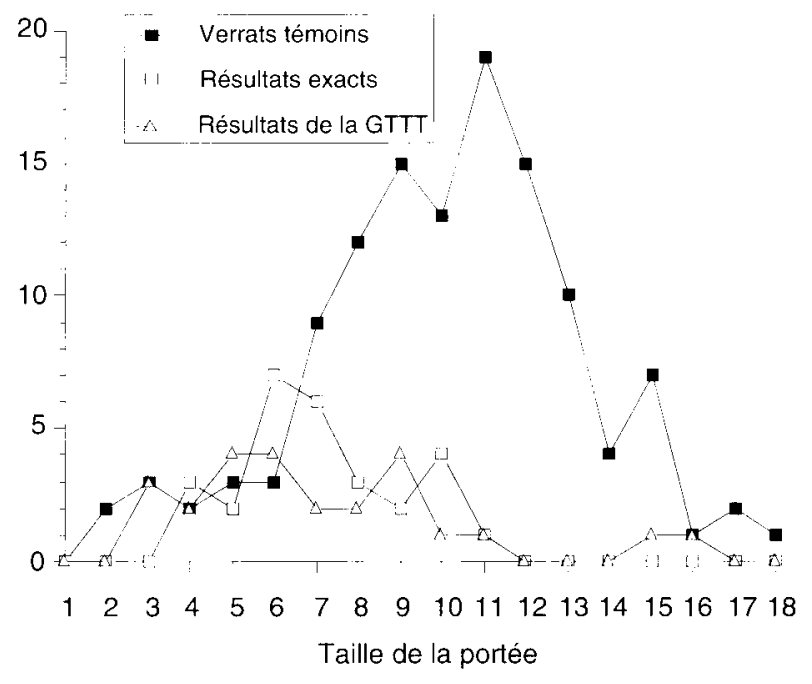

\section{2 / Les conséquences des translocations réciproques sur la reproduction}

Chez la truie les translocations réciproques n'ont pas d'effet significatif sur l'intervalle sevrage-oestrus (Bonneau 1990) ni sur la taux d'ovulation (figures 3 et 4). En revanche, selon la même étude, la présence d'une translocation chez le mâle ou la femelle augmente significativement l'intervalle sevrage-fécondation (figure 4).

La réduction de la taille de portées (figure 5, Bonneau et al 1991) des verrats porteurs de translocations est provoquée par la formation de gamètes à 
Figure 3. Nombre d'ovules pondus et nombre de porcelets à la naissance suivant le croisement pratiqué..

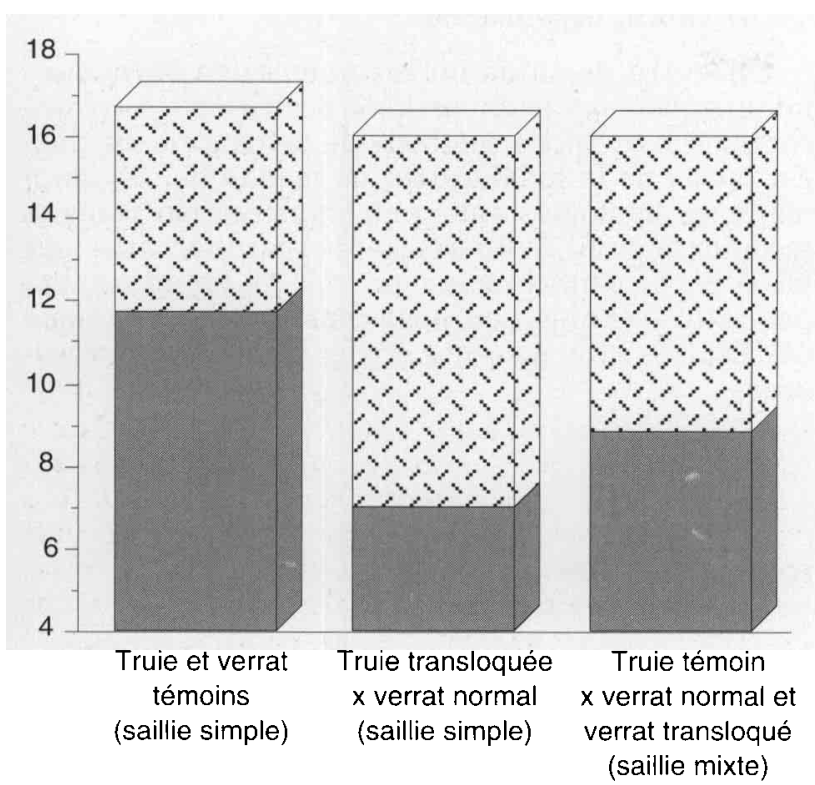

Nés vivants $\quad \because \quad$ Taux d'ovulation

Figure 4. Intervalles sevrage-cestrus (saillie) et sevragefécondation suivant le croisement pratiqué.

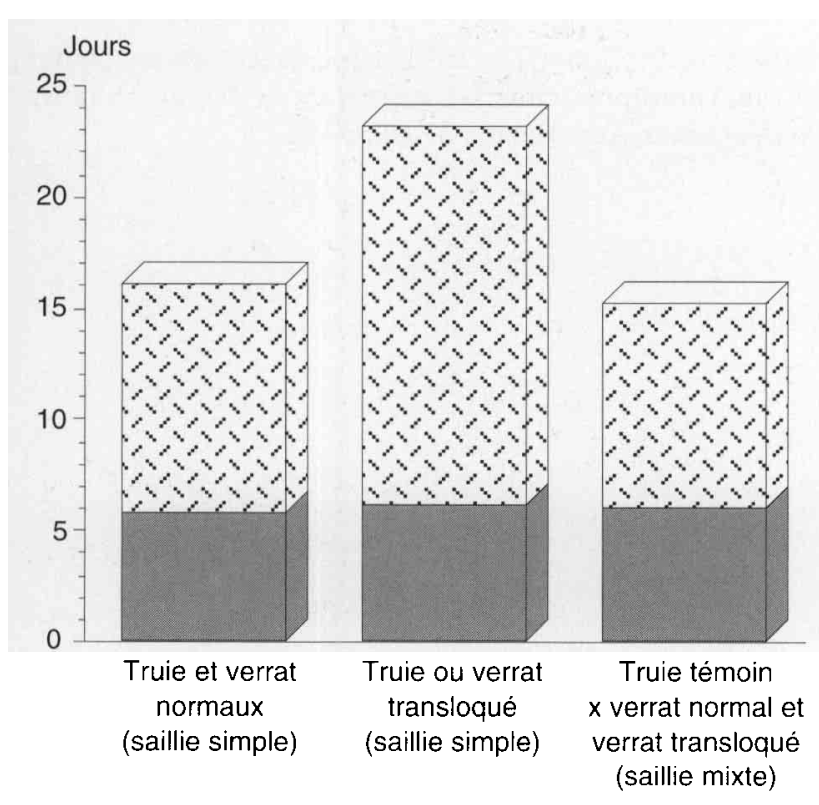

Intervalle sevrage - $\quad \because \quad$ Intervalle sevrage œstrus caryotype déséquilibré. En effet, un verrat porteur d'une translocation produit trois types de gamètes: normaux, porteurs de la translocation équilibrés (sans perte ni gain de matériel chromosomique) et déséquilibrés, auxquels il manque du matériel chromosomique ou, au contraire, il en existe en excès.
Figure 5. Distribution des tailles de portées conçues par les verrats porteurs, en comparaison aux verrats contemporains du même troupeau.
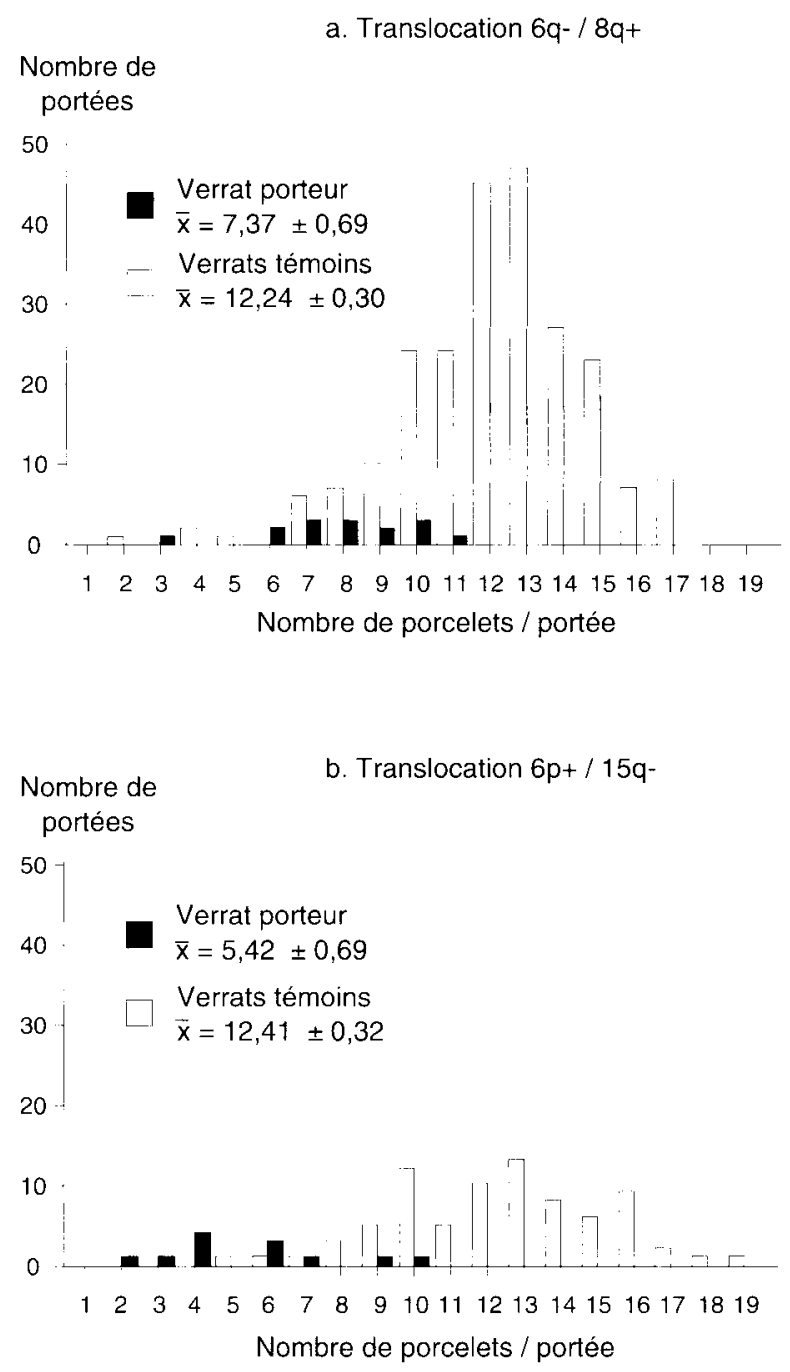

Les gamètes normaux assurent la persistance d'animaux normaux dans la population. Les gamètes porteurs de la translocation à l'état équilibré transmettent l'anomalie d'une génération à l'autre. Les gamètes déséquilibrés fécondent, ou sont fécondés normalement, mais produisent après la fécondation des embryons non viables qui sont éliminés avant ou pendant l'implantation.

Récemment, Gustavsson a signalé des caryotypes déséquilibrés par excès ou par défaut d'un segment chromosomique, chez des porcelets qui devaient mourir quelques heures après la naissance, issus d'un verrat porteur de la translocation 14/15.

Les conséquences économiques d'une translocation portée par un verrat peuvent varier suivant que ce dernier est en service chez un sélectionneur, un multiplicateur de cochettes ou de verrats terminaux ou enfin chez un producteur naisseur ou naisseurengraisseur. Rappelons que selon une estimation de Popescu et Tixier (1984) cette incidence représentait un manque à gagner de l'ordre de $48000 \mathrm{FF}$ chez un naisseur-engraisseur. La situation est plus grave lorsque le verrat est en service dans un centre d'insémination artificielle. 
Selon une simulation appliquée à un verrat utilisé en IA, le préjudice économique varie de 300000 à 600 $000 \mathrm{FF}$ selon qu'il s'agit d'un verrat de race pure ou de croisement (Bonneau 1990).

\section{3 / Quelques prolongements vers des recherches plus avancées}

Les anomalies chromosomiques peuvent avoir un rôle positif dans la cytogénétique des animaux de ferme. Elles représentent, en effet, un outil précieux pour les travaux de cartographie génique, étant utili- sées comme marqueurs. Un premier exemple pour le Porc a été l'utilisation de la translocation $3 / 7$ pour la localisation du système majeur d'histocompatibilité (SLA) (Geffrotin et al 1984).

Un autre domaine où les anomalies chromosomiques peuvent jouer un rôle positif est le tri des chromosomes par la méthode de cytométrie en flux. En raison de la modification de leur taille, les chromosomes impliqués dans une translocation peuvent modifier le profil de fluorescence et faciliter ainsi leur tri. A partir d'un chromosome isolé en grand nombre par cette technique on peut constituer une banque d'ADN, très utile pour des études de biologie moléculaire.

\section{Références bibliographiques}

Bonneau M., 1990. Translocations réciproques. Conséquences chez le porc domestique. Thèse de Diplôme de Recherche de l'Université PARIS XI.

Bonneau M., Boscher Jeannine, Delate J.J., Legault C., Popescu C.P., 1991. Deux nouvelles translocations réciproques impliquant le chromosome $6 \mathrm{chez}$ le porc domestique. Ann. Génét., 34, (2), 65-69.

Dagorn J., 1978. Note aux établissements Départementaux de l'Elevage. Institut Technique du Porc, Paris.

Gefrotin C., Popescu C.P., Cribiu E.P., Boscher Jeannine, Renard C., Chardon P., Vaiman C., 1984. Assignement of MHC in swine to chromosome 7 by in situ hybridization and serological typing. Ann. Génét., 27, (4), 213-219.

Legault C., Owen J., 1976. Etablissement de facteurs de correction de la taille de la portée pour l'âge de la mère à la mise bas dans les races porcines françaises. Journées de la Recherche Porcine en France 193-200.

Legault C., Molénat M., Steier G., Texier C., Lickler G., 1971. Principe et illustration d'un programme d'interprétation mécanographique des performances d'élevage des truies. In Journées de la Recherche Porcine en France 1971, 11-14, INRA-ITP 2D. Paris.

Popescu C.P., Legault C., 1979. Une nouvelle translocation réciproque $\mathrm{t}(4 \mathrm{q}+: 4 \mathrm{q})$, chez le porc domestique. Ann. Génét. Sél. Anim., 11, 361-369.

Popescu C.P., Tixier M., 1984. L'incidence des anomalies chromosomiques chez les animaux de ferme et leurs conséquences. Ann. Génét., 27, (2), 69-72. 